\title{
Dall'AIGA ad AQUA (con la collaborazione di ANIPA)
}

Marco Petitta - Presidente del Comitato Italiano IAH - marco.petitta@uniroma1.it

Daniela Ducci - Segretario del Comitato Italiano IAH - segretario@iahitaly.it

Tra gli eventi di interesse idrogeologico tenutisi nel 2015 spicca sicuramente in ambito nazionale il $\mathrm{V}$ Congresso dell' A.I.G.A. (Associazione Italiana di Geologia Applicata e Ambientale), svoltosi a Cagliari presso la Facoltà di Ingegneria dal 29 al 30 Aprile 2015, con il patrocinio dell'IAH Italia.

Le tematiche hanno riguardato i temi della Geologia Applicata e della Geologia Ambientale nell'accezione più ampia del termine, ma più del $40 \%$ dei lavori proposti sia come presentazioni che come poster si riferivano alle risorse idriche sotterranee, cui sono state dedicate due intere sessioni (Fig.1).

I temi trattati hanno spaziato dalla geotermia alla determinazione dei valori di fondo. Si è parlato anche molto di metodologie geofisiche e modellistica, oltre a vari studi idrogeologici locali.

Durante il Convegno c'è stato anche un intervento del Presidente Petitta sul progetto Kindra (Knowledge Inventory for bydrogeology research), progetto triennale finanziato dall'Unione Europea nell'ambito di Horizon2020, che si propone la creazione di un inventario delle conoscenze in campo idrogeologico, portando la sua testimonianza su un progetto europeo innovativo nell'ambito della gestione delle risorse idriche sotterranee.

L'eccellente organizzazione scientifica del Convegno da parte del socio Gabriele Uras è stata anche completata dall'ottima cena sociale tenutasi in un ristorante del lungomare del Poetto, durante la quale i convegnisti hanno avuto e la possibilità di confrontarsi sia dal punto di vista scientifico che conviviale.
In occasione del convegno di Cagliari si sono svolte anche le elezioni per il rinnovo della cariche istituzionali AIGA (Presidente, Consiglio Direttivo e Collegio dei Revisori), che hanno visto quattro soci IAH nominati Consiglieri per il triennio 2015-2018 (Marina De Maio, Walter Dragoni, Maurizio Polemio e Michele Saroli). Sono inoltre stati nominati Componenti del Collegio dei Revisori i Soci i soci IAH Francesco Fiorillo e Pantaleone De Vita.

Dal 25 al 29 maggio si è invece tenuta a Ustroń in Polonia, l'International Conference on "Groundwater Vulnerability - From Scientific Concept to Practical Application" (Fig.2). I colleghi polacchi, e in particolare il Prof. Andrzej Witkowski, hanno organizzato una conferenza di alto livello scientifico, con la partecipazione di 64 studiosi e ricercatori provenienti da molti paesi europei e anche da oltreoceano (Canada, USA, Australia, Sudafrica, Nigeria e Giappone). In un ambiente amichevole e stimolante dal punto di vista scientifico, la delegazione italiana, forte di 5 membri, è risultata la più numerosa dopo $i$ padroni di casa. Le sessioni scientifiche, partendo dal concetto di "vulnerabilità delle falde", hanno trattato gli aspetti metodologici proponendo nuove metodologie, le relazioni tra vulnerabilità e rischio, la valutazione di vulnerabilità in diversi contesti idrogeologici, etc. L'elevato livello scientifico è stato arricchito dalla partecipazione di esperti di fama nel settore della vulnerabilità delle falde quali Bartolomé Andreo-Navarro dell'Università di Malaga in Spagna, Alain Dassargues, dell’Università di Liegi in Belgio, Michael Sinreich dell’Uni-

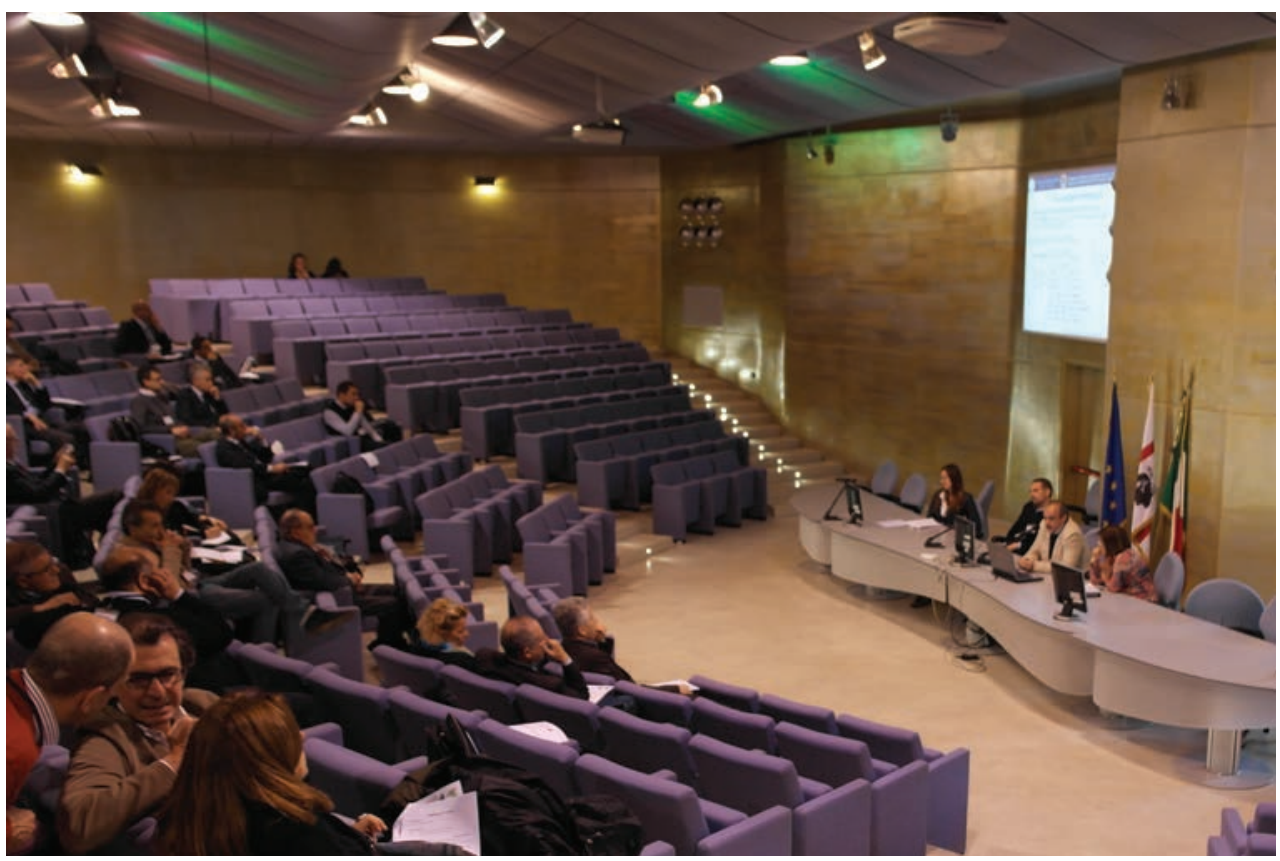

Fig. 1: Il congresso AIGA di Cagliari", foto di Laura Foddis. 


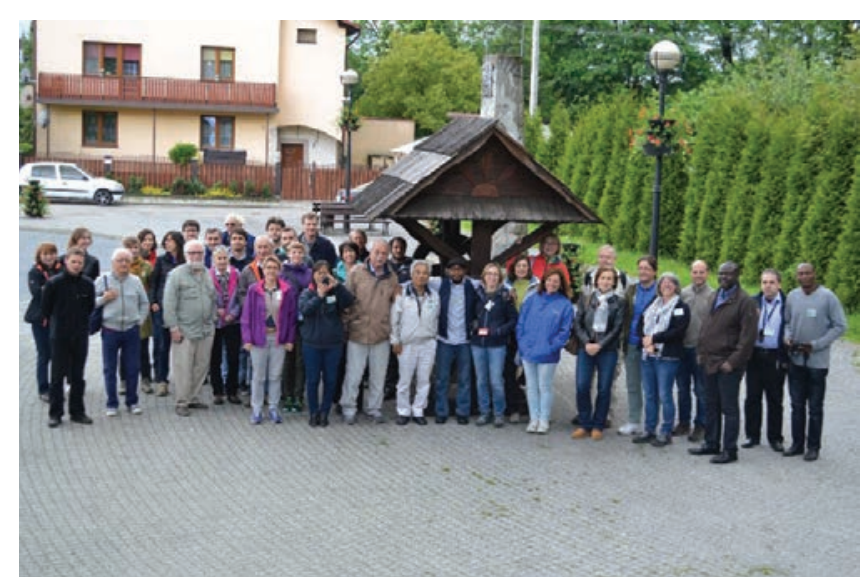

Fig. 2: Il field-trip dell'International Conference on "Groundwater Vulnerability - From Scientific Concept to Practical Application".

versità di Neuchâtel, e da altri stimati ricercatori come John Molson e James Lamoreaux, editor di Environmental Earth Sciences. Tutti i partecipanti hanno alloggiato presso la sede del convegno, un hotel della zona termale e sciistica dei monti Tatra nel sud della Polonia, e ciò ha favorito la discussione, lo scambio di esperienze e risultati, instaurando inoltre le basi per possibili future collaborazioni tra diversi paesi e ricercatori. Il field-trip di metà convegno e le numerose occasioni conviviali, quali un fantastico barbecue (nonostante la pioggia battente) e la cena di gala (con balli che hanno coinvolto anche il segretario IAH John Chilton...) hanno contribuito a rendere il clima partecipativo e accogliente. Il penultimo giorno si è tenuta l'assemblea IAH, nella quale siamo stati invitati a illustrare AQUA2015, con grande interesse da parte dei numerosi presenti, la maggior parte dei quali ha assicurato la propria presenza a Roma.

A proposito di AQUA2015, leggerete queste pagine quando ormai il Congresso sarà alle porte ed è quindi opportuno darvi qualche anticipazione. Tenendo conto del motto del congresso "Back to the future", il Comitato Organizzatore sta lavorando per offrire un evento indimenticabile, durante il quale da un lato si svilupperanno iniziative parallele alle classiche sessioni scientifiche (classificate come side event e workshop), dall'altro si cercherà di modernizzare quanto più possibile l'approccio standard dei congressi IAH.

Infatti, nella nuova sede della Città Universitaria della Sapienza (in Aula Magna e nel Dipartimento di Scienze della Terra), tutte le aule verranno fornite di connessione wireless, non tanto per dare l'opportunità ai partecipanti di leggere le mail e rispondere ai vari messaggi, ma perché, come ormai accade per altri grandi congressi come l'EGU di Vienna, ci si possa collegare al nuovo sito del congresso (www.aqua2015. com) dove il programma scientifico verrà aggiornato e reso consultabile on-line. In altre parole, verrà ridotto al minimo indispensabile il supporto cartaceo (un programma tascabile e poco altro), incrementando la possibilità di aggiornarsi on- line su cosa stia accadendo e cosa sia previsto nei giorni del congresso. Si mormora che anche il classico "gadget", che viene incluso normalmente della borsa congressuale, sarà in linea con la crescente necessità di connessione che interessa non solo ricercatori e professionisti, ma anche l'intera società a scala globale.

D'altronde, il programma si preannuncia talmente ampio da richiedere un'attenta analisi giorno per giorno se non ora per ora. Verranno attivate 7 sessioni orali parallele, che copriranno gli 8 temi principali con oltre 40 sessioni e più di 400 presentazioni, distribuite nell'arco della settimana. La sessione poster, come ormai noto, sarà per la prima volta in forma elettronica, con 6 postazioni dotate di schermi da 60" e tablet di consultazione, dove sarà possibile on-demand accedere a qualsiasi poster; inoltre, durante le 6 sessioni esclusivamente dedicate ai poster, gli Autori avranno a disposizione 4 minuti ciascuno per esporre oralmente i loro risultati; si prevedono oltre 500 poster elettronici, anche questi consultabili in rete per chi non vorrà spostarsi dalla propria sedia o non perdere il posto nella fila per il pranzo, che verrà servito sulla terrazza dietro l'Aula Magna. Un punto ristoro per i coffee break sarà presente anche nel Dipartimento di Scienze della Terra, dove si terranno le sessioni parallele.

L'Aula Magna verrà occupata non solo dalla cerimonia inaugurale con annessi eventi culturali, ma anche dalle 8 keynotes ognuna dedicata ad un diverso topic congressuale. Ma si terranno lì anche workshop tematici e eventi collaterali (side events), quali la presentazione del rapporto UNESCO WWAP sulle risorse idriche, l'esposizione di progetti europei Horizon2020 dedicati al tema acqua, la presentazione curata da ECHN insieme all'Hydrogeology Journal, e altre iniziative a cura degli sponsor, tra cui spicca la collaborazione con ANI$\mathrm{PA}$, cui è dedicata la giornata del venerdì. In quella occasione, con traduzione simultanea, verrà sviluppato un workshop sulla perforazione dei pozzi, al quale sarà possibile iscriversi anche separatamente, per facilitare la partecipazione dei professionisti geologi o comunque operanti nel mondo delle acque sotterranee, con riconoscimento dei crediti APC e quota di iscrizione giornaliera dedicata ai soci IAH ed ANIPA italiani. Altri eventi a cura di altri sponsor (quali UNESCO GRAPHIC e ESRI-ITALIA) e di gruppi di lavoro IAH Italia sono in cantiere!

Non vanno poi dimenticate le iniziative più collaudate, dai 5 corsi pre-congresso, ai tour post-congressuali, fino alla classica uscita tecnica di metà settimana, durante la quale verranno raggiunte 8 diverse mete distribuite nel Lazio e oltre, ma dando anche la possibilità per chi volesse restare a Roma di assistere all'Udienza Papale. E poi gli eventi sociali: il welcome cocktail la domenica, la serata dedicata ai giovani ECHN il lunedì, la cena nel centro storico il martedì e infine la cena sociale il giovedì durante la quale saremo ospiti di EATALY.

Veramente molto, forse troppo... L'obiettivo che ci siamo posti è quello di accontentare tutti, sia quelli che privilegiano gli aspetti scientifici che quelli più attenti alla socializzazione. Per riuscirci abbiamo bisogno della vostra presenza e del vostro parere. Vi aspettiamo tutti a Roma, dal 13 al 18 settembre! 